\title{
ANÁLISE DA RELAÇÃO ENTRE A ESTATURA E O PERÍMETRO ABDOMINAL EM INDIVÍDUOS PORTADORES DE PERCENTUAIS NORMAIS DE GORDURA
}

\author{
Relationship between the stature and abdominal circumference in individuals with normal fat \\ percentages
}

\author{
Walter Celso de LIMA, Ricardo Wallace das Chagas LUCAS, Paulo Afonso Nunes NASSIF, \\ Denise Serpa BOPP, Osvaldo MALAFAIA
}

Trabalho realizado na Universidade do Estado de Santa Catarina - UDESC, Florianópolis, SC, Brasil.

DESCRITORES - Obesidade. Adiposidade abdominal. Síndrome metabólica

\section{Correspondência:}

Ricardo Wallace das Chagas Lucas, e-mail: uniwallace@uniwallace.com.br

Fonte de financiamento: não há Conflito de interesses: não há

Recebido para publicação: 11/08/2009 Aceito para publicação: 14/01/2010

HEADINGS - Obesity. Abdominal adiposity. Metabolic syndrome
RESUMO - Racional - No indivíduo portador de obesidade há evidentes alterações morfofuncionais, que caracterizam a síndrome. São empregados vários parâmetros e índices perimétricos na análise de suas medidas antropométricas, que possuem relação com as alterações endocrinometabólicas. Muitos destes índices são aplicados de forma generalizada à população de uma maneira geral. Objetivo - Verificar a existência de uma razão cintura-estatura comum em indivíduos masculinos, de 18 a 25 anos de idade, portadores de percentuais de gordura normais, para fornecer modalidade de medida da cintura (perímetro) abdominal personalizada e não generalizada. Métodos - Foram selecionados de forma não probabilística intencional 380 sujeitos do sexo masculino, da faixa etária entre 18 e 45 anos, estratos de estatura de 160 a 169 cm, 170 a $179 \mathrm{~cm}$ e de 180 a 189 cm, do banco de dados do Ambulatório da Clínica Escola da empresa IBRATE - Instituto Brasileiro de Therapias e Ensino, sede de Curitiba/PR, com dados inclusos entre os anos de 2004 a 2008. Desta população foram triados 174 indivíduos, da faixa etária entre 18 e 25 anos, possuidores de percentuais de gordura dentro da faixa de normalidade, ou seja, entre $4 \%$ e $16 \%$ de gordura. Foi determinado coeficiente de correlação de Pearson ( $r$ ) entre o percentual de gordura e o perímetro abdominal; foi determinada a média e o desvio-padrão de todas as variáveis dispostas, e o intervalo de confiança para 95\% para média do perímetro abdominal e as razões cintura-estatura da população foram transcritos em percentuais, e determinadas as médias do perímetro abdominal. Resultados - A análise da amostra de 174 indivíduos resultou estarem na faixa etária de 21,2 + 2,1 anos; com estatura de 174,3 +6,2 cm; com percentual de gordura de 10,8 \%; com medida do perímetro abdominal de $75,5+5,7 \mathrm{~cm}$ e com a razão cintura/estatura apresentando o valor de $0,43+0,033$. Conclusão - Existe relação comum da razão cintura-estatura entre indivíduos do sexo masculino de faixa etária entre 18 e 25 anos portadores de percentual normal de gordura de $43 \%$ do valor de sua estatura.
ABSTRACT - Background - Obese people have evident morphologic changes characterizing the syndrome. Different parameters and index are used to analyze their anthropometric measurements related to metabolic diseases. Many of these are applied widely to the population in general. Aim - To verify the existence of the waist-height common in male individuals from 18 to 25 years of age, with normal percentages of fat, to provide a method for measuring the circumference (perimeter) abdominal. Methods - It was selected a nonprobabilistic intentional 380 male subjects group, aged between 18 and 45 years (2004 to 2008) with160 to $169 \mathrm{~cm}, 170$ to $179 \mathrm{~cm}$ and 180 to $189 \mathrm{~cm}$ of height, taken from the database Outpatient Clinic School Company IBRATE - Brazilian Institute of Therapy and Education, based in Curitiba, PR, Brasil. From this population were screened 174 individuals, aged between 18 and 25 years, owners of percentage of fat within the normal range between $4 \%$ and $16 \%$. Spearman's correlation coefficient ( $r$ ) was used between fat percentage and waist circumference, determined by mean and standard deviation of all variables, with confidence interval of $95 \%$ for average waist circumference and population reasons waist-height. Results - The analysis showed 174 men with age 21,2 + 2,1 y; height $174,3+6,2 \mathrm{~cm}$; fat percentage of $10,8 \%$; abdominal circumference of $75,5+5,7 \mathrm{~cm}$ and waist-height reason of $0,43+0,033$. Conclusion - There is a correlation in the waist-height among men from 18 and 25 years old with normal fat percentage of $43 \%$ of the stature. 


\section{INTRODUÇÃO}

E xiste aceitação nos estudos epidemiológicos da utilização de medidas antropométricas fixas para homens e mulheres em relação à cintura abdominal. Os limites de corte utilizados apresentam números absolutos que estão em acordo com tipo de população avaliada ${ }^{4}$. Dentre os dados levantados para compor as análises de risco cardiometabólico, a estatura do indivíduo por ser relativamente imutável após idade adulta e tem servido como base para a composição de medidas mais aplicáveis às diversas populações ${ }^{15}$.

Em relação à medida do perímetro (cintura) abdominal, questiona-se a adequação do ponto de corte estabelecido em $102 \mathrm{~cm}$ para homens e $88 \mathrm{~cm}$ para mulheres ${ }^{4}$ para populações de diferentes etnias. Pois em alguns estudos, níveis mais baixos, tais como $94 \mathrm{~cm}$ para homens e $80 \mathrm{~cm}$ para mulheres, têm sido considerados mais apropriados 2,12,14,18.

A Diretriz Brasileira de Diagnóstico e Tratamento da Síndrome Metabólica recomenda monitorização mais frequente dos fatores de risco para doenças coronarianas, para mulheres com circunferência (perímetro) de cintura abdominal entre 80 e $88 \mathrm{~cm}$ e para homens entre 94 e102 $\mathrm{cm}$. Alguns estudos internacionais estabeleceram que a obesidade é fator de risco de doença cardiovascular estatisticamente significativo, em basicamente todas as populações do mundo. O Interheart constituiu-se de um estudo caso-controle padronizado, com 262 centros participantes em 52 países da África, Ásia, Austrália, Europa, Oriente Médio, América do Norte e América do Sul, que trouxe como resultado um total de 15.152 casos incidentes de infarto agudo do miocárdio (IAM) e 14.820 controles pareados por idade ( \pm 5 anos) e sexo, mas sem história de doença cardíaca ${ }^{20}$.

As análises subsequentes previamente estabelecidas dos resultados do Interheart determinaram adicionalmente que o cálculo da razão cintura-quadril é um fator preditivo mais poderoso do nível de risco de doença cardiovascular associado à obesidade que qualquer outra medida de obesidade, como o IMC - Índice de Massa Corporal por exemplo, ou grupo de medidas ${ }^{3}$.

O estudo IDEA (Dia Internacional para Avaliação da Obesidade Abdominal, no original em inglês International Day for the Evaluation of Abdominal Obesity), também demonstrou que o perímetro abdominal é melhor preditor de resultados das doenças cardiovasculares que o IMC. Os primeiros resultados deste estudo de mais de 170.000 pessoas indicam que a medida abdominal está associada às doenças cardiovasculares, independentemente da relação que o índice de massa corporal tenha com o risco de doença cardiovascular, sem considerar idade ou geografia.

Os dados do estudo IDEA foram apresentados na $55^{a}$ Sessão Científica Anual do Colégio Americano de
Cardiologia realizada em 2006, em Atlanta, Geórgia. Segundo Haffner, et al. ${ }^{3}$, confirma-se a importância de medir a circunferência abdominal, junto com medidas atuais como o índice de massa corporal, pressão sanguínea, glicemia e níveis lipídicos, para identificar pacientes que estão sob crescente risco cardiometabólico em cenário de cuidados primários.

O estudo IDEA envolveu 6.407 médicos clínicos gerais em 63 países (excluindo os Estados Unidos). Uma população não selecionada composta por 177.345 pacientes com idades de 18 a 80 anos que consultaram seu médico clínico geral por qualquer razão em duas meias jornadas previamente estabelecidas foram incluídos no estudo entre maio e julho do ano de 2005. Além das medidas da circunferência abdominal, altura e massa corporal total (peso) corporal, foram recolhidos dados demográficos por gênero, idade e nível de instrução. A presença ou ausência de fatores de risco clássicos de doença cardiovascular (por exemplo, hipertensão, diabete, dislipidemia e tabagismo) e os fatores de risco existentes (doença cardíaca coronariana, acidente vascular encefálico ou revascularização prévia) também foram registrados. As características relevantes da população IDEA foram: 1) predomínio de sobrepeso (ponto de corte de índice de massa corporal $25-30 \mathrm{~kg} / \mathrm{m}^{2}$ ) não diferiu regionalmente, mas foi consistentemente maior em homens que em mulheres; 2) predomínio de obesidade (índice de massa corporal $\geq 30 \mathrm{~kg} / \mathrm{m}^{2}$ ) foi muito alto de maneira geral, tendo sido o mais alto no Canadá e nos países do Oriente Médio, e mais baixo na Ásia; 3) medida da circunferência abdominal tanto em homens quanto em mulheres foi mais baixa no Leste Asiático e mais alta na África do Sul, Oriente Médio e Leste Europeu.

A prevalência mundial de doença cardiovascular diagnosticada por médicos em homens no estudo foi de $16 \%$, variando de $10 \%$ na América Latina a $26 \%$ no Leste Europeu. Nas mulheres, a prevalência mundial foi de $12,5 \%$, variando de $7 \%$ na América do Norte a $23 \%$ no Leste Europeu.

A análise dos dados demonstrou que tanto o índice de massa corporal como o perímetro abdominal estavam associados, independentemente da presença de doença cardiovascular. Cada aumento na circunferência abdominal de $14 \mathrm{~cm}$ para os homens e $14,9 \mathrm{~cm}$ para as mulheres aumentava a probabilidade de uma pessoa apresentar doença cardiovascular entre $21 \%$ e $40 \%$. Quando ajustada para o índice de massa corporal, a medida abdominal era fator preditivo ainda mais forte de doença cardiovascular, particularmente nos homens.

As relações independentes com as doenças cardiovasculares para o perímetro abdominal e o índice de massa corporal foram vistos em todas as regiões geográficas, mesmo naquelas onde as pessoas eram magras, tais como no Leste Asiático. Entretanto, em 75\% das regiões, a associação das doenças cardiovasculares com o perímetro abdominal foi mais forte que a associação com o índice de massa corporal. 
O estudo europeu da EPIC (Investigação Prospectiva Européia em Câncer e Nutrição) examinou a associação do IMC, circunferência da cintura e relação cintura-quadril com o risco de morte entre 359.387 participantes de nove países. Foram usadas análises de regressão, com a idade como a variável tempo, os modelos e estratificada de acordo com o centro de estudos e idade no recrutamento, com ajuste adicional para o nível educacional, tabagismo, consumo de álcool, atividade física e altura. Neste estudo durante seguimento médio de 9,7 anos, 14.723 participantes morreram. O menor risco de morte relacionada ao IMC foi observado em IMC de 25,3 para os homens e 24,3 para as mulheres. Após o ajuste para o IMC, circunferência da cintura e relação cintura-quadril foram fortemente associados com o risco de morte. Riscos relativos entre os homens e mulheres apresentaram-se no quintil mais alto da circunferência da cintura. IMC permaneceu significativamente associado com o risco de morte nos modelos que incluíam a circunferência da cintura e relação cintura-quadril $(P<0,001)$.

Os dados sugerem que tanto a adiposidade geral e a adiposidade abdominal estão associadas com o risco de morte, assim deve-se apoiar a utilização de perímetros da cintura abdominal e da relação cinturaquadril, além de IMC na avaliação do risco de morte.

Ainda é citado que diferentes etnias apresentam diferentes somatotipos, e consequentemente distribuições de gordura diversas, de modo que valores de corte preditivos de risco em população, podem não ser válidos para todas.

Em vista do exposto, abre-se lacuna que possibilita a criação hipotética de uma forma de medição da circunferência (perímetro) abdominal, que esteja mais relacionada com população específica, demonstrando pontos de corte menos estandardizados, como atualmente se apresentam. Para tanto, abre-se o pressuposto que em corpos adultos saudáveis, existem proporções comuns entre as partes. Utilizando a estatura como a parte relativamente imutável, variações superiores ou inferiores, devem se diferenciar em relação ao gênero, somatotipo e faixa etária.

Desta forma o objetivo deste estudo é verificar a existência de uma razão cintura-estatura comum em indivíduos masculinos, de 18 a 25 anos de idade, portadores de percentuais de gordura normais, para fornecer modalidade de medida da cintura (perímetro) abdominal mais flexível e aplicável nos estudos epidemiológicos.

\section{MÉTODOS}

Este é estudo descritivo correlacional, onde o objeto é a verificação da existência de relação percentual comum de razão cintura-estatura em indivíduos portadores de percentuais de gordura normais ${ }^{17}$.

A população consta do banco de dados do ambulatório da clínica-escola da empresa IBRATE Instituto Brasileiro de Therapias e Ensino, sede de Curitiba, PR, com dados inclusos entre os anos de 2004 a 2008. O projeto foi aprovado pelo Comitê de Ética da mesma instituição sob protocolo número 037/09.

Este banco de dados oferece dados de: idade, estatura, perímetro abdominal e percentual de gordura dos indivíduos. Foram selecionados de forma não-probabilística intencional 380 sujeitos do sexo masculino, da faixa etária entre 18 e 45 anos. Desta população foram triados 174 indivíduos, da faixa etária entre 18 e 25 anos, possuidores de percentuais de gordura dentro da faixa de normalidade, ou seja, entre $4 \%$ e $16 \%$ de gordura (Figura 1 ).

\begin{tabular}{|c|c|c|c|c|c|c|}
\hline \multicolumn{7}{|c|}{ PERCENTUAL DE GORDURA X IMC (HOMENS) } \\
\hline \multicolumn{2}{|c|}{ Nível/Idade } & $18-25$ & $26-35$ & $36-45$ & $46-55$ & $56-65$ \\
\hline $\begin{array}{c}\text { Atlético/ } \\
\text { Risco }\end{array}$ & $=$ ou $<12 \mathrm{~kg} / \mathrm{m}^{2}$ & $\left(^{*}\right) 4$ a $7 \%$ & $\left({ }^{\star}\right) 8$ a $11 \%$ & (*) 10 a $15 \%$ & 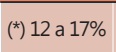 & (*) 13 a $19 \%$ \\
\hline Atlético & $12.1<18.5 \mathrm{Kg} / \mathrm{m}^{2}$ & 8 a $11 \%$ & 12 a $15 \%$ & 16 a $18 \%$ & 18 a $20 \%$ & 20 a $21 \%$ \\
\hline Ótimo & $18.6<19.9 \mathrm{Kg} / \mathrm{m}^{2}$ & 12 a $13 \%$ & 16 a $18 \%$ & 19 a $21 \%$ & 21 a $23 \%$ & 22 a $23 \%$ \\
\hline Saudável & $20<24.9 \mathrm{Kg} / \mathrm{m}^{2}$ & 14 a $16 \%$ & 19 a $21 \%$ & 22 a $24 \%$ & 24 a $25 \%$ & 24 a $25 \%$ \\
\hline Sobrepeso & $25<29.9 \mathrm{Kg} / \mathrm{m}^{2}$ & 17 a $20 \%$ & 22 a $24 \%$ & 25 a $27 \%$ & 26 a $27 \%$ & 26 a $27 \%$ \\
\hline $\begin{array}{l}\text { Obeso } \\
\text { Grau I }\end{array}$ & $30<34.9 \mathrm{Kg} / \mathrm{m}^{2}$ & 21 a $24 \%$ & 25 a $27 \%$ & 28 a $30 \%$ & 28 a $31 \%$ & 28 a $31 \%$ \\
\hline $\begin{array}{l}\text { Obeso } \\
\text { Grau II }\end{array}$ & $35<39.9 \mathrm{Kg} / \mathrm{m}^{2}$ & 26 a $36 \%$ & 28 a $36 \%$ & 31 a $39 \%$ & 32 a $38 \%$ & 32 а 38\% \\
\hline \multicolumn{7}{|c|}{ PERCENTUAL DE GORDURA X IMC (MULHERES) } \\
\hline \multicolumn{2}{|c|}{ Nível/Idade } & $18-25$ & $26-35$ & $36-45$ & $46-55$ & $56-65$ \\
\hline $\begin{array}{l}\text { Atlético/ } \\
\text { Risco }\end{array}$ & $<12 \mathrm{~kg} / \mathrm{m}^{2}$ & $\left(^{*}\right) 13$ a $16 \%$ & $\left(^{*}\right) 14$ a $17 \%$ & 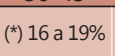 & 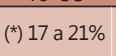 & (*) 18 a $22 \%$ \\
\hline Atlético & $12.1<18.5 \mathrm{Kg} / \mathrm{m}^{2}$ & 17 a $19 \%$ & 18 a $20 \%$ & 20 a $23 \%$ & 22 a $25 \%$ & 24 a $26 \%$ \\
\hline Ótimo & $18.6<19.9 \mathrm{Kg} / \mathrm{m}^{2}$ & 20 a $22 \%$ & 21 a $23 \%$ & 24 a $26 \%$ & 26 a $28 \%$ & 27 a $29 \%$ \\
\hline Saudável & $20<24.9 \mathrm{Kg} / \mathrm{m}^{2}$ & 23 a $25 \%$ & 24 a $25 \%$ & 27 a $29 \%$ & 29 a $31 \%$ & 30 a $32 \%$ \\
\hline Sobrepeso & $25<29.9 \mathrm{Kg} / \mathrm{m}^{2}$ & 25 a $28 \%$ & 27 a $29 \%$ & 30 a $32 \%$ & 32 a $34 \%$ & 33 a $35 \%$ \\
\hline $\begin{array}{l}\text { Obeso } \\
\text { Grau I }\end{array}$ & $30<34.9 \mathrm{Kg} / \mathrm{m}^{2}$ & 29 a $31 \%$ & 30 a $33 \%$ & 33 а $36 \%$ & 35 а $38 \%$ & 36 a $38 \%$ \\
\hline $\begin{array}{l}\text { Obeso } \\
\text { Grau II }\end{array}$ & $35<39.9 \mathrm{Kg} / \mathrm{m}^{2}$ & 32 a $43 \%$ & 34 a $49 \%$ & 37 a $48 \%$ & 39 a $50 \%$ & 39 a $49 \%$ \\
\hline
\end{tabular}

Referencial: Pollock e Wilmore, 1993'16; e OMS, $1997^{19}$

FIGURA 1 - Percentual de gordura x índice de massa corpórea (IMC) em homens e mulheres

O banco de dados possui o conjunto das variáveis coletadas, que foram mensuradas utilizando os seguintes instrumentos: estatura - através de estadiômetro portátil constituído por escala métrica com resolução de $1 \mathrm{~mm}$; massa corporal total - balança digital portátil calibrada, com capacidade para $150 \mathrm{~kg}$, com margem de $100 \mathrm{~g}$; perímetro abdominal - através de fita métrica inextensível, com margem de 0,1 cm; percentual de gordura - através do equipamento de bioimpedância bipolar da marca Omron, modelo HBF-306.

Todos os dados foram transportados para planilha do Microsoft Excel 2007. Para a coleta dos dados da população pesquisada foram realizados os seguintes procedimentos, em sequência: estatura, perímetro abdominal, percentual de gordura e razão cintura-estatura

A estatura foi mensurada com indivíduo em pé e com pés descalços, trajando calção ou sunga com recomendação dos seguintes procedimentos para o dia e antes do exame: a) não comer ou beber por quatro horas; b) não fazer exercícios físicos não habituais por 12 horas; c) urinar 30 minutos antes; d) não consumir álcool 
por 48 horas; e) não fazer uso de diuréticos por sete dias; f) massa corporal total (peso) em pé e com descalço.

O perímetro abdominal foi feito com o posicionamento da fita métrica em pontos anatômicos recomendados pela I Diretriz Brasileira de Diagnóstico e Tratamento da Síndrome Metabólica e da OMS (Organização Mundial de Saúde) medida, no meio da distância entre a crista ilíaca e o rebordo costal inferior, com o indivíduo de pé ${ }^{9,10,11}$.

Para o percentual de gordura foram incorporados os dados de sexo, idade, massa corporal total e nível de condicionamento (ser ou não ser atleta) com o indivíduo em pé e calçado no aparelho. Para tratamento dos dados foi utilizado o programa estatístico do Excel, incluído no Software Microsoft 2007. Foram realizados procedimentos estatísticos com os dados, após adicioná-los em colunas no Excel 2007, dispostos na sequência de idade, estatura, perímetro abdominal, percentual de gordura e razão cinturaestatura. Foram utilizados o coeficiente de correlação de Pearson (r), média e o desvio-padrão de todas as variáveis dispostas, e o intervalo de confiança para 95\% para média do perímetro abdominal. As razões cintura-estatura da população foram transcritas em percentuais, e determinadas as médias do perímetro abdominal.

\section{RESULTADOS}

A análise da amostra de 174 indivíduos resultou estarem eles na faixa etária de 21,2 + 2,1 anos; com estatura de 174,3 +6,2 cm; com percentual de gordura de $10,8 \%$; com medida do perímetro abdominal de 75,5 $+5,7 \mathrm{~cm}$ e com a razão cintura/estatura apresentando o valor de 0,43+0,033 (Tabela 1 ).

TABELA 1 - Média e desvios-padrão de todas as variáveis dispostas $(n=174)$

\begin{tabular}{cccccc}
\hline & IDADE & ESTATURA & $\begin{array}{c}\text { PERÍMETRO } \\
\text { ABDOMINAL }\end{array}$ & $\begin{array}{c}\text { \% DE } \\
\text { GORDURA }\end{array}$ & $\begin{array}{c}\text { RAZÃO } \\
\text { CINTURA }\end{array}$ \\
ESTATURA
\end{tabular}

alpha $0,05 /$ intervalo de confiança $=0.840$

\section{DISCUSSÃO}

A correlação positiva muito forte $(r$ de Pearson $=0,778$ ) entre o percentual de gordura e o perímetro abdominal corrobora com a assertiva que quanto maior o percentual de gordura maior o perímetro da cintura ${ }^{1}$. E quando a correlação foi ajustada para o percentual de gordura e a razão cinturaestatura também se verificou correlação positiva muito forte. Isto demonstra que na população heterogênea masculina estudada, a relação cintura-estatura pode ser preditor de aumento ou diminuição de gordura abdominal (visceral) ${ }^{5,6}$.

Quando selecionada a amostra, a necessidade de realizarem-se as correlações de cintura-estatura com percentual de gordura deixou de ser o foco. Isto é claro quando analisa-se a baixa correlação positiva entre o percentual de gordura e o perímetro abdominal ( $r$ de Pearson $=0,373$ ) - amostra é composta de indivíduos com percentuais de gordura normais e homogêneos -, perde-se o objetivo de avaliar o aumento ou diminuição desta variável, e suas implicações consequentes à gordura visceral ${ }^{7,8}$.

Realizando a comparação da média da razão cintura-estatura da população total, com a amostra, observa-se aumento percentual significativo no desvio-padrão, apesar de não se observar diretamente este aumento pelas diferenças absolutas das médias. A hipótese levantada para esta situação pode ser em função da diferença do " $n$ " amostral.

Dividindo a amostra por estratos de faixa de estatura, observa-se que não há diferença significativa entre as razões cintura-estatura, e utilizando-se o intervalo de confiança de $95 \%$ da média amostral observa-se que a existência da amplitude maior de diferença está relacionada ao " $n$ " já que o alpha é o mesmo e não há diferença significativa entre os desviospadrão. Desta forma, nesta população a utilização da média geral das razões cintura-estatura apresenta-se como um parâmetro suficiente para analisar-se o valor ideal da medida da cintura (perímetro) abdominal, sem levar em consideração a estatura dos indivíduos (IC0,840).

Como a soma total dos indivíduos da população estudada, assim como os indivíduos escolhidos para compor a amostra apresentaram percentual de gordura médio baixo em relação à tabela de referência, e em consequência também da baixa razão cintura-estatura, pode-se inferir que a população e os indivíduos da amostra apresentaram baixo risco cardiometabólico.

Reportando aos estudos de Hsieh, et al. ${ }^{7}$ e de Pitanga, et al. ${ }^{13}$, que sugerem que o valor do perímetro abdominal, de forma prática, não deve exceder $50 \%$ da estatura - os valores da razão cintura-estatura para este fim são em torno de 0,5 -, pode-se inferir que o presente estudo apresentou concordância com eles. A diferença é aqui ocorreu caminho inverso em relação aos pontos de corte que determinam o limite superior para a medida do perímetro abdominal, ou seja, partiu-se do princípio que indivíduos portadores de percentuais de gordura normais, deveriam manter relações métricas comuns e homogêneas. Desta forma o ponto de corte buscado seria para os limites normais, e não anormais e desencadeadores de distúrbios cardiometabólicos

Com isto, a partir da amostra estudada, pode-se sugerir que existe uma faixa limite de ação para os pontos de corte para a razão cintura-estatura, representado entre 0,43 e 0,50, que traduzido para a linguagem de 
percentual, seria entre $43 \%$ e $50 \%$ da estatura do indivíduo avaliado, guardando as devidas proporções de faixa etária e gênero, sendo que quanto mais baixa a idade, menor pode ser o percentual de gordura e consequentemente menor perímetro abdominal, desde que se respeitem os parâmetros de massa corporal total ideal. Assim, inferese que quanto mais a medida do perímetro abdominal se afastar do limite inferior, maiores serão as chances de desenvolvimento de distúrbios cardiometabólicos.

Novos estudos devem ser estimulados, a partir da idéia de alcançar faixas etárias maiores e menores, grupos étnicos populacionais diferentes, e levantar o peso da razão cintura-estatura como instrumento de aplicação na saúde pública, quando aplicados junto aos demais marcadores cardiometabólicos.

\section{CONCLUSÃO}

Existe relação comum da razão cintura-estatura entre indivíduos do sexo masculino de faixa etária entre 18 e 25 anos portadores de percentual normal de gordura de $43 \%$ do valor de sua estatura.

\section{REFERÊNCIAS}

1. Callegari-Jacques SM. Bioestatística. Princípios e Aplicações.Porto Alegre, ArtMed, 2003.

2. Ferreira MG, Valente JG, Gonçalves-Silva RMV, Sichieri R. Acurácia da circunferência da cintura e da relação cintura/quadril como preditores de dislipidemias em estudo transversal de doadores de sangue de Cuiabá, Mato Grosso, Brasil. Cad Saúde Pública, 2006; 22(2):307-14.

3. Haffner SM, Despres JP, Balkau B. Waist circumference and body mass index are both independently associated with cardiovascular disease: The International Day for the Evaluation of Abdominal Obesity (IDEA) survey. J Am Coll Cardiol. 2006;47(4 suppl A):358A. Abstract 842-6.

4. Han TS, van Leer EM, Seidell JC, Lean ME. Waist circumference action levels in the identification of cardiovascular risk factors: prevalence study in a random sample. BMJ. 1995 Nov 25;311(7017):1401-5.

5. Hsieh SD, Yoshinaga H, Muto T, Sakurai Y, Kosaka K. Health risks among Japanese men with moderate body mass index. Int J Obes Relat Metab Disord. 2000 Mar;24(3):358-62.

6. Hsieh SD, Yoshinaga H, Muto T, Sakurai Y. Anthropometric obesity indices in relation to age and gender in Japanese adults. Tohoku J
Exp Med. 2000 Jun;191(2):79-84.

7. Hsieh SD, Yoshinaga H, Muto T, Sakurai Y. Regular physical activity and coronary risk factors in Japanese men. Circulation. 1998 Feb 24;97(7):661-5..

8. Hsieh SD, Yoshinaga H. Do people with similar waist circumference share similar health risks irrespective of height? Tohoku J Exp Med. 1999 May;188(1):55-60.

9. I Diretriz brasileira de Diagnóstico e Tratamento da Síndrome Metabólica. Rev. da Sociedade Brasileira de Hipertensão, 2004:4:122-62.

10. Iribarren C, Darbinian JA, Lo JC, Fireman BH, Go AS. Value of the sagittal abdominal diameter in coronary heart disease risk assessment: cohort study in a large, multiethnic population. Am J Epidemiol. 2006 Dec 15;164(12):1150-9.

11.IV Diretriz brasileira sobre Dislipidemias e Prevenção da Aterosclerose. Departamento de Aterosclerose da Sociedade Brasileira de Cardiologia. Arq Bras Cardiol. 2007;88(suppl. I).

12. Oh JY, Hong YS, Sung YA, Barrett-Connor E. Prevalence and factor analysis of metabolic syndrome in an urban Korean population. Diabetes Care. 2004 Aug;27(8):2027-32.

13. Pitanga FJG, Lessa I. Associação entre indicadores antropométricos de obesidade e risco coronariano em adultos na cidade de Salvador, Bahia, Brasil. Rev Bras Epidemiol. 2007;10(2):239-48.

14. Pitanga FJG, Lessa I. Indicadores antropométricos de obesidade como instrumento de triagem para risco coronariano elevado em adultos na cidade de Salvador - Bahia. Arq Bras Cardiol. 2005;85(1):26-31.

15. Pitanga FJG, Lessa I. Razão cintura-estatura como discriminador de risco coronariano de adultos. Rev Assoc Med Bras. [online] 2006;52(3).

16. Pollock ML, Wilmore JH. saúde

ício na Saúde e na Doença, $2^{\mathrm{a}}$ ed., Editora Médica e Científica Ltda. Rio de Janeiro - RJ, 1993.

17. Thomas JR, Nelson JK. Métodos de Pesquisa em Atividade Física. Porto Alegre. Artmed, 2002.

18. Vasquez ACJ, Rosado LEFPL, Rosado GP, Ribeiro RCL, Franceschini SCC, Geloneze B, Priore SE, Oliveira DR. Habilidade de indicadores antropométricos e de composição corporal em identificar a resistência à insulina. Arq Bras Endocrinol Metab.2009;53(1):72-9.

19. WHO - World Health Organization. Obesity: preventing and managing the global epidemic. Report of a WHO consultation on obesity. Geneve:World Health Organization; 1997.

20. Yusuf S, Hawken S, Ounpuu S, Dans T, Avezum A, Lanas F, McQueen M, Budaj A, Pais P, Varigos J, Lisheng L. Effect of potentially modifiable risk factors associated with myocardial infarction in 52 countries (the INTERHEART study): case-control study. Lancet. 2004 Sep 11-17:364(9438):937-52. 